\title{
Spin-torque diode with tunable sensitivity and bandwidth by out-of-plane magnetic field
}

\author{
X. Li, ${ }^{1}$ C. Zheng, ${ }^{1}$ Y. Zhou, ${ }^{2,3, a)}$ H. Kubota, ${ }^{4}$ S. Yuasa,${ }^{4}$ and Philip W. T. Pong ${ }^{1}$ \\ ${ }^{1}$ Department of Electrical and Electronic Engineering, The University of Hong Kong, Hong Kong \\ ${ }^{2}$ School of Electronics Science and Engineering, Nanjing University, Nanjing 210093, China \\ ${ }^{3}$ Department of Physics, The University of Hong Kong, Hong Kong \\ ${ }^{4}$ Spintronics Research Center, National Institute of Advanced Industrial Science and Technology, Tsukuba, \\ Ibaraki 305-8568, Japan
}

(Received 24 February 2016; accepted 23 May 2016; published online 8 June 2016)

\begin{abstract}
Spin-torque diodes based on nanosized magnetic tunnel junctions are novel microwave detectors with high sensitivity and wide frequency bandwidth. While previous reports mainly focus on improving the sensitivity, the approaches to extend the bandwidth are limited. This work experimentally demonstrates that through optimizing the orientation of the external magnetic field, wide bandwidth can be achieved while maintaining high sensitivity. The mechanism of the frequency- and sensitivity-tuning is investigated through analyzing the dependence of resonant frequency and DC voltage on the magnitude and the tilt angle of hard-plane magnetic field. The frequency dependence is qualitatively explicated by Kittel's ferromagnetic resonance model. The asymmetric resonant frequency at positive and negative magnetic field is verified by the numerical simulation considering the in-plane anisotropy. The DC voltage dependence is interpreted through evaluating the misalignment angle between the magnetization of the free layer and the reference layer. The tunability of the detector performance by the magnetic field angle is evaluated through characterizing the sensitivity and bandwidth under 3D magnetic field. The frequency bandwidth up to $9.8 \mathrm{GHz}$ or maximum sensitivity up to $154 \mathrm{mV} / \mathrm{mW}$ (after impedance mismatch correction) can be achieved by tuning the angle of the applied magnetic field. The results show that the bandwidth and sensitivity can be controlled and adjusted through optimizing the orientation of the magnetic field for various applications and requirements. Published by AIP Publishing.
\end{abstract}

[http://dx.doi.org/10.1063/1.4953572]

The spin-transfer torque (STT) of direct current will excite radio-frequency (RF) oscillation in nanosized magnetic tunneling junctions (MTJs), leading to the emergence of spintorque oscillators. ${ }^{1}$ On the other hand, it has also been demonstrated that RF signal can be rectified into DC voltage according to the spin-torque diode effect in MTJ nanopillars, ${ }^{2}$ revealing the potential application as microwave detectors. ${ }^{3,4}$ When the alternating current (AC) flows through a MTJ nanopillar, the free-layer magnetization oscillates around its equilibrium state, resulting in variable resistance of the device at the $\mathrm{AC}$ frequency. The $\mathrm{AC}$ is thus mixed with the alternating resistance, leading to a frequency-dependent DC voltage $\left(V_{m i x}\right)$. Largest DC voltage $\left(V_{D C}\right)$ is generated when the ferromagnetic resonant frequency of free layer $\left(f_{R}\right)$ matches that of the applied microwave. The sensitivity of this microwave detector is evaluated as Sensitivity $=V_{D C} / P_{R F}$. The frequency bandwidth is illustrated as the bandwidth of achievable $f_{R}$ within the available magnetic field range, since $f_{R}$ can be tuned by the magnitude of magnetic field. The recent report of sensitivity of over $12000 \mathrm{mV} / \mathrm{mW}$ (Ref. 5) and $75400 \mathrm{mV} / \mathrm{mW}$ (Ref. 6) for microwave detection has exceeded that of Schottky diode detectors, showing its great promise for real applications.

In the past decade, efforts have been devoted to increasing the $V_{D C}$ and sensitivity. Previous experimental investigations

\footnotetext{
${ }^{\text {a) }}$ Author to whom correspondence should be addressed. Electronic mail: yanzhou@hku.hk
}

have revealed that the $V_{D C}$ can be increased by applying a DC bias, ${ }^{5}$ by optimizing the in-plane (IP) magnetic field angle, ${ }^{7}$ and by adjusting the alignment between the magnetization of the reference layer and the free layer. ${ }^{8,9}$ It has also been theoretically predicted that $V_{D C}$ can be optimized by adjusting the orientation of the out-of-plane (OOP) magnetic field. ${ }^{10}$ Although several experimental reports show high sensitivity under oblique magnetic field with certain angles, ${ }^{5,11}$ there still lacks a systematic experimental investigation of the dependence of $V_{D C}$ on the tilt angle of OOP magnetic field. The bandwidth, on the other hand, is constrained by the available magnetic field range since $f_{R}$ is primarily determined by the magnitude of external magnetic field $\left(H_{\text {ext }}\right)$. The introduction of tilt magnetic field offers an additional degree of freedom for extending the frequency bandwidth according to the angular dependence of $f_{R}{ }^{12}$ Experimental demonstration on how the $V_{D C}$ and $f_{R}$ can be tuned by the magnitude and tilt angle of OOP magnetic field will be beneficial for improving the performance of spin-torque-diode microwave detectors.

In this work, the spin-torque diode effect in MTJ nanopillar has been investigated under OOP magnetic field. First, the magnetic field is applied in the hard plane, and the dependence of the $V_{D C}$ and $f_{R}$ on the magnitude and tilt angle is analyzed. Extended bandwidth and increased sensitivity are later demonstrated through tuning the orientation of the magnetic field.

The device under investigation is a $200 \times 100 \mathrm{~nm}^{2}$ elliptical MTJ nanopillar with a layer structure of Ta 5/CuN 20/Ta $3 /$ 
PtMn $\quad 15 / \mathrm{Co}_{70} \mathrm{Fe}_{30} \quad 2.5 / \mathrm{Ru} \quad 0.85 / \mathrm{Co}_{60} \mathrm{Fe}_{20} \mathrm{~B}_{20} \quad 3 / \mathrm{MgO} \sim 1 /$ $\mathrm{Co}_{60} \mathrm{Fe}_{20} \mathrm{~B}_{20}$ 2/Ta 5/Ru 7 (thickness in nm). When IP magnetic field is applied parallel to the easy axis, the MTJ exhibits parallel resistance-area product of $19 \Omega \mu \mathrm{m}^{2}$ and tunnel magnetoresistance (TMR) ratio of 34\% (Fig. 1(a)). The experimental setup for spin-torque diode experiment is shown in Fig. 1(b). Microwave power of $-30 \mathrm{dBm}(1 \mu \mathrm{W})$ is pulse-modulated at $8 \mathrm{kHz}$ and injected into the MTJ through a bias-tee. The $V_{\text {mix }}$ is measured by a lock-in amplifier when the frequency of microwave current ramps from $2 \mathrm{GHz}$ to $12 \mathrm{GHz}$. The output power of RF synthesizer is flattened to compensate for the larger loss at higher frequencies. In each measurement, an external magnetic field is applied with polar angle $\left(\theta_{H}\right)$ and azimuthal angle $\left(\delta_{H}\right)$ (Fig. 1(a) inset) to align the magnetization of free layer (m) at $\left(\theta_{F}, \delta_{F}\right)$.

First, we explore how $V_{D C}$ and $f_{R}$ are tuned by the magnitude of hard-plane magnetic field $\left(\delta_{H}=90^{\circ}\right)$. The magnetoresistance (MR) curves measured at $\theta_{H}=0^{\circ}-90^{\circ}$ are shown in Fig. 2(a). Under different $H_{e x t}$, the misalignment angle $(\varphi)$ between $\mathbf{m}$ and the magnetization of reference layer $(\mathbf{M})$ is calculated based on Eq. (1) $)^{5}$ and shown in Fig. 2(b).

$$
R^{-1}(\varphi)=\frac{R_{\mathrm{AP}}^{-1}+R_{P}^{-1}}{2}-\frac{R_{\mathrm{AP}}^{-1}-R_{P}^{-1}}{2} \cos \varphi,
$$

where $R(\varphi)$ is the junction resistance, $R_{A P}$ and $R_{P}$ are the resistances at antiparallel and parallel state, respectively. The anisotropy $\left(H_{K}\right)$ exhibits a shape anisotropy $\left(H_{S A}\right)$ at $x$ axis, an out-of-plane anisotropy $\left(H_{O A}\right)$ originated from the $\mathrm{MgO} / \mathrm{CoFeB} / \mathrm{Ta}$ interfaces,${ }^{13-15}$ and a fourfold magnetocrystalline anisotropy $\left(H_{C A}\right)^{16}$ of the partially crystallized $\mathrm{CoFeB}$ after annealing. ${ }^{17}$ The offset angle $(\beta)$ between the crystalline easy axis and $x$-axis results in the asymmetric resistances at negative and positive $H_{e x t}$. When large positive $H_{e x t}$ is applied at $\theta_{H}=45^{\circ}, \mathbf{m}$ is rotated to the first octant ( 1 in Fig. 2(b)), while $\mathbf{M}$ shifts a small IP angle towards $y$-axis. This results in a misalignment angle of $\varphi=50^{\circ}$. As positive $H_{\text {ext }}$ decreases to $0, \mathbf{m}$ and $\mathbf{M}$ gradually resume the initial alignment of $\varphi=20^{\circ}$ (2 in Fig. 2(b)). Under increasing negative $H_{\text {ext }}, \mathbf{m}$ is rotated to the seventh octant due to the fourfold $H_{C A}$ (3 in Fig. 2(b)), resulting in the maximum $\varphi$ of $85^{\circ} . \mathbf{m}$ is aligned back towards the $y-z$ plane when negative $\mu_{0} H_{e x t}$ is further increased to $-0.15 \mathrm{~T}$, leading to decreasing $\varphi$ towards $60^{\circ}$. It is noted that the resistance and $\varphi$ change little in the field range of $-0.15-0.15 \mathrm{~T}$ with $\theta_{H}=90^{\circ}$. This is explained by the small OOP angle of $\mathbf{m}$ when $H_{e x t}$ is much

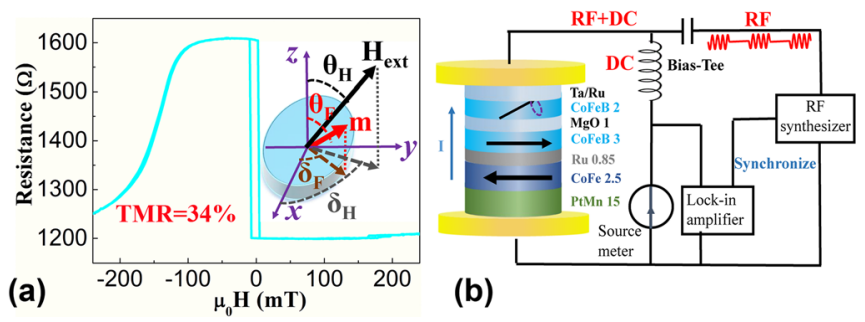

FIG. 1. (a) MR curve measured at $\theta_{H}=90^{\circ}$ and $\delta_{H}=0^{\circ}$ (inset: polar and azimuthal angles of $H_{\text {ext }}$ and $\mathbf{m}$, where $x$ is the easy axis and $z$ is perpendicular to the film plane). (b) Experimental setup for measuring the spin-torque diode spectrum. smaller than the demagnetization field. In this case, $\varphi$ is nearly unchanged within the field range.

The changing $\varphi$ with $H_{\text {ext }}$ results in different RF rectification responses. The $V_{m i x}$-frequency spectra measured at $\mu_{0} H_{\text {ext }}$ ranging from $-0.15 \mathrm{~T}$ to $0.15 \mathrm{~T}$ with $\theta_{H}=60^{\circ}$ are shown in Fig. 2(c). A typical $V_{m i x}$-frequency spectrum is composed of a symmetric Lorentzian peak and an antiLorentzian component. The contribution of Lorentzian peak is much larger than the anti-Lorentzian component, indicating the IP component of STT plays a dominant role. ${ }^{16}$ The larger $\varphi$ in negative field results in more IP STT component, so the peaks are higher and more symmetric compared with the peaks at positive field. To evaluate the sensitivity and bandwidth of the detector, the dependences of $V_{D C}$ and $f_{R}$ on $H_{\text {ext }}$ with $\theta_{H}=0^{\circ}-90^{\circ}$ are shown in Figs. 2(d) and 2(e), respectively. The $V_{D C}$ in a symmetric MTJ can be expressed as ${ }^{7}$

$$
V_{D C}=\frac{R_{A P}-R_{P}}{R_{P}} \frac{\mu_{B}}{2 e\left(m_{S} t A\right) \sigma} \frac{P}{1+P^{2}} \frac{R^{2}}{R_{P} R_{\mathrm{AP}}} \frac{P_{R F}^{2}}{4} \sin ^{2} \varphi,
$$

where $m_{S}, t, A$, and $P$ are the saturation magnetization, thickness, area, and polarization ratio of the free layer, respectively, and $\sigma$ is the linewidth of the resonant peak. The expression in Eq. (2) is consistent with the experimental results in Fig. 2(d) that $V_{D C}$ reaches maximum at the magnetic field where MR and $\varphi$ are also largest. The V-shape dependence of $f_{R}$ on negative $H_{e x t}$ with $\theta_{H}=0^{\circ}-90^{\circ}$ and $\delta_{H}=90^{\circ}$ in Fig. 2(e) can be interpreted qualitatively based on the ferromagnetic resonance model proposed by Kittel ${ }^{18,19}$

$$
f_{R}=\frac{\gamma}{2 \pi} \sqrt{H_{e f f m}\left(H_{e f f m}+H_{d}\right)}
$$

in which $\gamma=1.76 \times 10^{7} \mathrm{~Hz} / \mathrm{Oe}$ is the gyromagnetic ratio of electrons, $H_{d}$ is the demagnetization field, and $H_{\text {effm }}$ is the effective field, representing the combined effect of $H_{\text {ext }}$ and $H_{K}$ on $\mathbf{m}$. At small $H_{e x t}, H_{K}$ is counteracted by the IP component of $H_{e x t}$, resulting in a reduction in $H_{\text {effm }}$ with increasing $H_{e x t}$. So, $f_{R}$ tends to decrease as $H_{\text {ext }}$ increases. The minimum $f_{R}$ indicates that the free layer is saturated by the IP component of $H_{\text {ext }}{ }^{20}$ As $H_{\text {ext }}$ further increases, $H_{\text {effi }}$ becomes larger and $f_{R}$ increases accordingly. $H_{C A}$ is responsible for the asymmetric $f_{R}-H_{e x t}$ relation at positive and negative $H_{e x t}$. An analytical model ${ }^{10,21}$ is used to quantitatively investigate this $f_{R}-H_{e x t}$ relation with $\theta_{H}=45^{\circ}$ and $\delta_{H}=90^{\circ}$. The magnetic free energy density $(F)$ of the free layer can be expressed as the summation of Zeeman energy $\left(F_{\text {Zeeman }}\right)$, demagnetization energy $\left(F_{\text {demag }}\right)$, uniaxial anisotropy energy $\left(F_{U A}\right)$, and crystalline anisotropy energy $\left(F_{C A}\right)$, defined, respectively, by ${ }^{21,22}$

$$
\begin{gathered}
F_{\text {Zeeman }}=-m_{S} H_{e x t}\left[\sin \theta_{H} \sin \theta_{F} \cos \left(\delta_{F}-\delta_{H}\right)\right. \\
\left.+\cos \theta_{H} \cos \theta_{F}\right], \\
F_{\text {demag }}+F_{U A}=\frac{1}{2} m_{S}\left[-H_{S A} \sin ^{2} \theta_{F} \cos ^{2} \delta_{F}\right. \\
\left.+\left(4 \pi m_{S}-H_{O A}\right) \cos ^{2} \theta_{F}\right], \\
F_{C A}=\frac{1}{8} m_{S} H_{C A}\left[\sin ^{2} 2 \theta_{F}+\sin ^{4} \theta_{F} \sin ^{2} 2\left(\delta_{F}-\beta\right)\right] .
\end{gathered}
$$



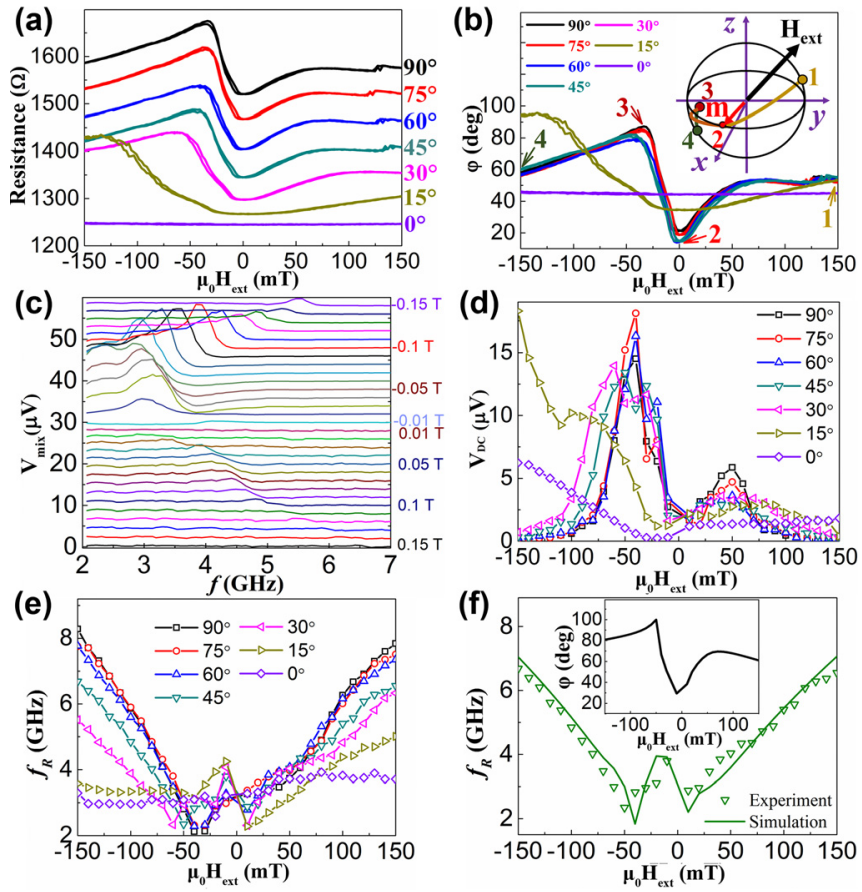

FIG. 2. Influence of magnitude and tilt angle $\left(\theta_{H}\right)$ of hard-plane magnetic field. (a) Hard-axis MR curves. (b) $\varphi$ calculated by Eq. (1) (inset: schematic path of the free-layer magnetization direction when $\mu_{0} H_{\text {ext }}$ is scanned from $0.15 \mathrm{~T}$ to $-0.15 \mathrm{~T}$ at $\theta_{H}=45^{\circ}$ ). (c) Spin-torque diode spectrum measured with $\theta_{H}=60^{\circ}$ and $\mu_{0} H_{\text {ext }}$ from 0.15 to $-0.15 \mathrm{~T}$. Each curve is shifted by $2 \mu \mathrm{V}$. (d) $V_{D C}$ and (e) $f_{R}$ measured as a function of $H_{\text {ext }}$. (f) Measured (triangle) and simulated (solid line) $f_{R}-H_{e x t}$ relation with $\theta_{H}=45^{\circ}$ (inset: simulated $\varphi-H_{\text {ext }}$ relation).

The equilibrium angles $\left(\theta_{F}, \delta_{F}\right)$ of $\mathbf{m}$ can be calculated from $\frac{\partial F}{\partial \delta_{F}}=0$ and $\frac{\partial F}{\partial \theta_{F}}=0$.

$$
\begin{aligned}
\frac{\partial F}{\partial \delta_{F}}= & m_{\mathrm{S}}\left[H_{e x t} \sin \theta_{H} \sin \theta_{F} \sin \left(\delta_{F}-\delta_{H}\right)\right. \\
& -\frac{1}{2} H_{S A} \sin 2 \delta_{F} \sin ^{2} \theta_{F} \\
& \left.-\frac{1}{4} H_{C A} \sin ^{4} \theta_{F} \sin 4\left(\delta_{F}-\beta\right)\right]=0,
\end{aligned}
$$

$$
\begin{aligned}
\frac{\partial F}{\partial \theta_{F}}= & m_{\mathrm{S}}\left\{H_{e x t}\left[\sin \theta_{H} \cos \theta_{F} \cos \left(\delta_{F}-\delta_{H}\right)-\cos \theta_{H} \sin \theta_{F}\right]\right. \\
& +2 \pi m_{\mathrm{S}}\left(1-\frac{H_{O A}}{4 \pi m_{\mathrm{S}}}\right) \sin 2 \theta_{F}+\frac{1}{2} H_{S A} \cos ^{2} \delta_{F} \sin 2 \theta_{F} \\
& \left.-\frac{1}{4} H_{C A}\left[\sin 4 \theta_{F}+2 \sin ^{3} \theta_{F} \sin ^{2} 2\left(\delta_{F}-\beta\right)\right]\right\}=0 .
\end{aligned}
$$

$f_{R}$ can thus be determined from ${ }^{23}$

$$
f_{R}=\frac{\gamma}{2 \pi} \frac{1}{m_{S}} \sqrt{\frac{\partial^{2} F}{\partial \theta_{F}^{2}} \frac{\partial^{2} F}{\partial \delta_{F}^{2}}-\left(\frac{\partial^{2} F}{\partial \theta_{F} \partial \delta_{F}}\right)^{2}} .
$$

The $f_{R}-H_{\text {ext }}$ relation at $\theta_{H}=45^{\circ}$ are well reproduced by the numerical simulation based on Eq. (9), as shown in Fig. 2(f). In the simulation, $m_{S}=808 \mathrm{emu} / \mathrm{cm}^{3}$ is characterized from DC magnetometry. $\mu_{0} H_{O A}=0.35 \mathrm{~T}, \mu_{0} H_{S A}=18 \mathrm{mT}, \mu_{O} H_{C A}=17 \mathrm{mT}$, and $\beta=55^{\circ}$ are determined from best fit. The small effective demagnetization field $\left(4 \pi \mu_{0} \mathrm{~m}_{\mathrm{S}}-\mu_{0} H_{O A}=0.65 \mathrm{~T}\right)$ is also possibly attributed to the finite junction size and the effect of dipolar field. ${ }^{24}$ It is noted that the experimental results show higher $f_{R}$ around the minimum $\left(\mu_{0} H=-50 \mathrm{mT}\right.$ or $10 \mathrm{mT})$. This can be explained by the higher $H_{\text {effm }}$ contributed by the edge effects and defects, resulting in higher $f_{R}$ according to Eq. (3). The validity of this model is further confirmed by the similar $\varphi-H_{\text {ext }}$ relation between the simulation (Fig. 2(f), inset) and the calculation based on experiment (Fig. 2(b)).

In order to further investigate the influence of the tilt angle of the hard-plane magnetic field, $f_{R}$ is plotted as a function of $\theta_{H}$ in Fig. 3(a). The measured $f_{R}$ exhibits a minimum at $\theta_{H}=10^{\circ}-15^{\circ}$, and then gradually increases at larger $\theta_{H}$. To reveal the mechanism behind this angular dependence, the equilibrium angles $\left(\theta_{F}, \delta_{F}\right)$ of $\mathbf{m}$ under the hard-plane magnetic field $\left(\mu_{0} H_{\text {ext }}=-0.1 \mathrm{~T}\right)$ tilted by different $\theta_{H}$ are calculated based on Eqs. (7) and (8) and shown in the right axis of Fig. 3(b). The $f_{R}-\theta_{H}$ relation is qualitatively interpreted by Eq. (3). At small $\theta_{H}$, the IP effective field is dominated by IP anisotropy. As $\theta_{H}$ increases from $0^{\circ}$ to $10^{\circ}-15^{\circ}$, $H_{\text {effm }}$ gradually decreases as the increasing IP component of $H_{\text {ext }}$ overshadows the anisotropy. Meanwhile, m rotates away towards the $y$-axis $\left(-\delta_{F}\right.$ increasing from $<40^{\circ}$ to $102^{\circ}$ in the right axis of Fig. 3(b)), resulting in declining $H_{\text {effm }}$ and thus decreasing $f_{R}$. When $\theta_{H}>15^{\circ}, \mathbf{m}$ is maintained near the $y$-axis. As $\theta_{H}$ increases, the angle between $H_{\text {ext }}$ and $\mathbf{m}$ decreases from $60^{\circ}$ to $\sim 0^{\circ}$. The $H_{\text {effm }}$ increases as the effect of $H_{\text {ext }}$ on $\mathbf{m}$ increases, leading to increasing $f_{R}$ with $\theta_{H}$. The numerical simulation based on Eq. (9) exhibits similar trend as compared to the experimental result at $\mu_{0} H_{e x t}=-0.1 \mathrm{~T}$ (Fig. 3(b), left axis). The difference in $f_{R}$ between the experiments and simulations when $\theta_{H}<10^{\circ}$ is believed to be resulted from the loss of single domain state under weak IP effective field. Further, the $f_{R}$ and $V_{D C}$ at different $H_{\text {ext }}$ and $\theta_{H}$ are shown in Figs. 3(c) and 3(d), respectively, to evaluate the influence of magnitude and direction of magnetic field. $f_{R}$ is higher under larger $H_{\text {ext }}$ and larger $\theta_{H}$. The minimum $f_{R}$ in (a)
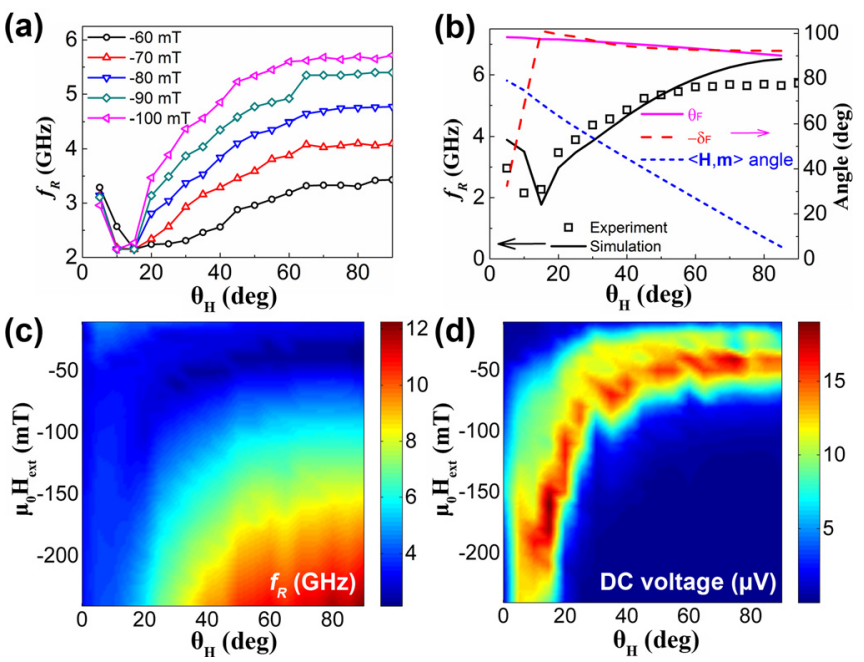

(d)

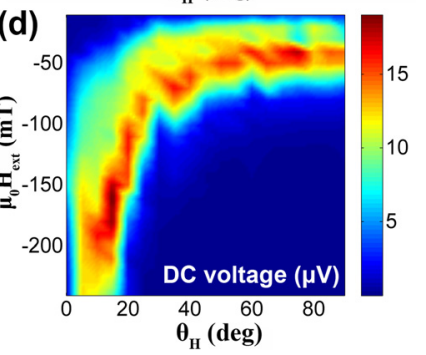

FIG. 3. Influence of $\theta_{H}$ when $\delta_{H}=90^{\circ}$ (a) $f_{R}$ as a function of $\theta_{H}$. (b) Calculated $\theta_{F}, \delta_{F}$ and angle between $H_{e x t}$ and $\mathbf{m}$ (right $y$-axis) and measured (square) and simulated (solid line) $f_{R}$ (left $y$-axis) as a function of $\theta_{H}$ with $\mu_{0} H_{e x t}=-100 \mathrm{mT}$. (c) $f_{R}$ and (d) $V_{D C}$ as a function of $H_{\text {ext }}$ and $\theta_{H}$. 
Fig. 3(c) coincides with the maximum $V_{D C}$ in Fig. 3(d). This can be interpreted by the maximum $\varphi$ when $\mathbf{m}$ is parallel to the hard axis. It is noted that at smaller $\theta_{H}, V_{D C}$ reaches maximum under larger $H_{\text {ext }}$. When $\theta_{H}=15^{\circ}$, the largest $V_{D C}$ of $19.9 \mu \mathrm{V}$ is achieved at $-160 \mathrm{mT}$. While at $\theta_{H}=60^{\circ}, V_{D C}$ reaches maximum of $16.3 \mu \mathrm{V}$ at $-40 \mathrm{mT}$. This is because when $\theta_{H}$ is smaller, larger $H_{\text {ext }}$ is required to provide sufficient IP component $\left(H_{\text {ext }} \sin \theta_{H}\right)$ to saturate the free layer. These results indicate that both the magnitude and the OOP angle of magnetic field are effective in tuning the $f_{R}$ and $V_{D C}$.

Finally, in order to evaluate how the performance of the spin torque diode detector can be tuned by the OOP angle of the magnetic field, the sensitivity and $f_{R}$ at $\theta_{H}=0^{\circ}-90^{\circ}$ and $\delta_{H}=30^{\circ}-90^{\circ}$ are characterized. $V_{D C}$ and $f_{R}$ at $\mu_{0} H_{\text {ext }}=-0.1 \mathrm{~T}$ are shown in Figs. 4(a) and 4(b), respectively. Higher $V_{D C}$ is achieved at larger $\delta_{H}$. This is because $V_{\text {mix }}$ is proportional to $\sin ^{2} \varphi$ (Eq. (2)), and larger $\varphi$ is expected when the magnetic field is applied towards the hard axis (large $\delta_{H}$ ). $V_{D C}$ reaches maximum at $\theta_{H}=20^{\circ}$ and $\delta_{H}=90^{\circ}$, accompanied with the minimum $f_{R}$. This can be explained by the smaller $H_{\text {effm }}$ when larger $\varphi$ exists. As interpreted in Fig. 3(d), $\theta_{H}$ corresponding to the maximum $V_{D C}$ gradually decreases with increasing $H_{\text {ext }}$. $f_{R}$ is relatively higher when $\theta_{H}$ approaches $90^{\circ}$. This is consistent with the observation that $f_{R}$ increases with $\theta_{H}$ in Fig. 3(a). The maximum sensitivity (Fig. 4(c)) and the frequency bandwidth (Fig. 4(d)) are defined as the highest sensitivity and the frequency range between the maximum and minimum $f_{R}$, respectively, when $\mu_{0} H_{\text {ext }}$ is changed from $-0.01 \mathrm{~T}$ to $-0.2 \mathrm{~T}$.
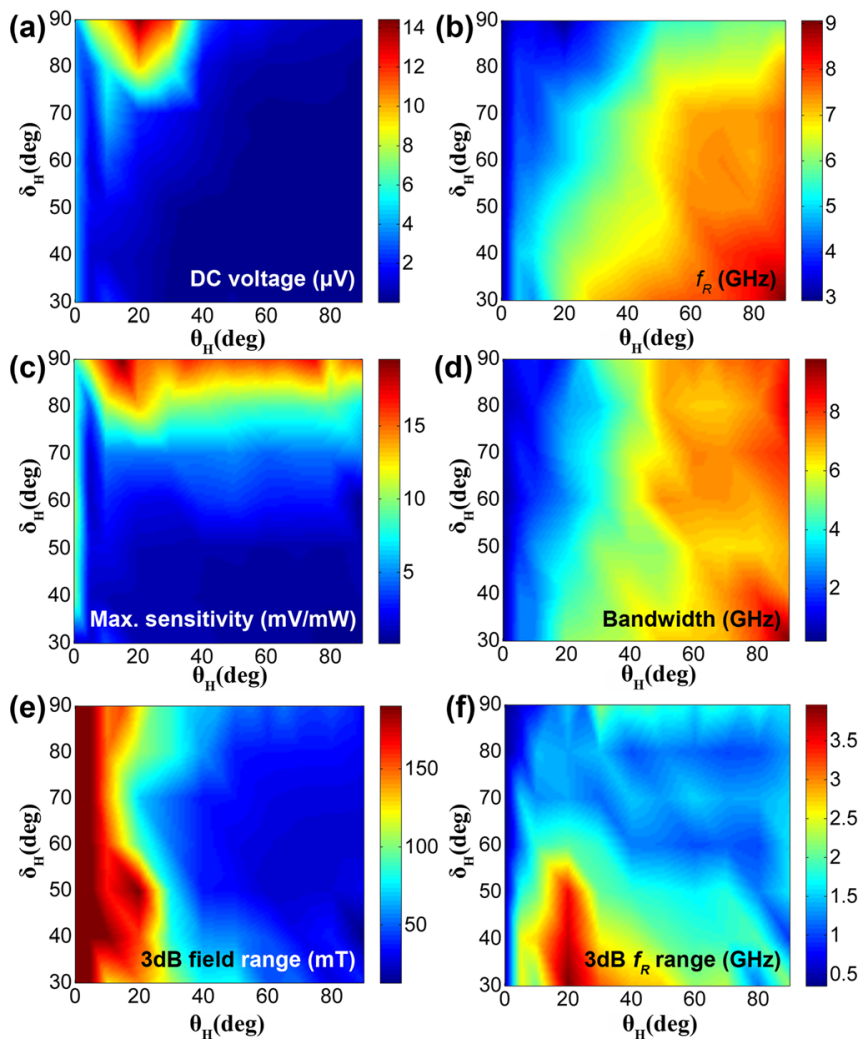

FIG. 4. Influence of the OOP angle of the magnetic field: (a) $V_{D C}$ and (b) $f_{R}$ under $\mu_{0} H_{\text {ext }}=-0.1 \mathrm{~T}$ and (c) the maximum sensitivity, (d) the frequency bandwidth, (e) the $3 \mathrm{~dB}$ field range, and (f) the $3 \mathrm{~dB} f_{R}$ range when $\mu_{0} H_{e x t}$ is swept from -0.01 to $-0.2 \mathrm{~T}$.
Similar to Fig. 4(a), high sensitivity is achieved at large $\delta_{H}$. The bandwidth is primarily determined by $f_{R}$ at $\mu_{0} H_{e x t}$ $=-0.2 \mathrm{~T}$, so Fig. 4(d) exhibits similar angular dependence with Fig. 4(b). The $3 \mathrm{~dB}$ field range and $f_{R}$ range are shown, respectively, in Figs. 4(e) and 4(f) to illustrate the range of $H_{\text {ext }}$ and $f_{R}$ where the sensitivity is higher than $50 \%(-3 \mathrm{~dB})$ of the maximum at a certain field angle. The $3 \mathrm{~dB}$ field range is larger at small $\theta_{H}$, due to the smaller $V_{d c}-H_{\text {ext }}$ dependence when the magnetic field rotates towards OOP, as shown in Fig. 2(d). However, the larger $3 \mathrm{~dB}$ field range when $\theta_{H}$ approaches $0^{\circ}$ only results in small $3 \mathrm{~dB} f_{R}$ range. This can be illustrated by the low field dependence of $f_{R}$ at small $\theta_{H}$, as shown in Fig. 2(e). Through combined analysis of the maximum sensitivity, bandwidth, and $3 \mathrm{~dB} f_{R}$ range, the performance of the microwave detector over the full field range can be evaluated. The optimized maximum sensitivity of $19.9 \mathrm{mV} /$ $\mathrm{mW}$ (or $151 \mathrm{mV} / \mathrm{mW}$ after correction of impedance mismatch) is observed at $\theta_{H}=15^{\circ}$ and $\delta_{H}=90^{\circ}$. This indicates $29 \%$ increase in sensitivity compared with the case of IP magnetic field $\left(15.7 \mathrm{mV} / \mathrm{mW}\right.$ at $\theta_{H}=90^{\circ}$ and $\left.\delta_{H}=90^{\circ}\right)$. The bandwidth of $1.1 \mathrm{GHz}$ and $3 \mathrm{~dB} f_{R}$ range of $1 \mathrm{GHz}$ indicates that high sensitivity is granted in most of the frequency band. On the other hand, widest bandwidth of $9.8 \mathrm{GHz}$ is achieved when $\theta_{H}=90^{\circ}$ and $\delta_{H}=30^{\circ}$, and this bandwidth is expected to be further extended when $\delta_{F}$ is smaller than $30^{\circ}$. However, the corresponding low maximum sensitivity $(0.11 \mathrm{mV} / \mathrm{mW})$ and $3 \mathrm{~dB} f_{R}$ range $(2.24 \mathrm{GHz})$ indicate poor microwave detectivity. Although the co-existence of wide $3 \mathrm{~dB} f_{R}$ range and high sensitivity remains to be difficult, a compromised performance can be achieved by fine-tuning the tilt angle of the magnetic field. For example, the direction of the magnetic field can be set to be $\theta_{H}=30^{\circ}$ and $\delta_{H}=90^{\circ}$ to achieve a bandwidth of $4.49 \mathrm{GHz}$ and maximum sensitivity of $14.7 \mathrm{mV} / \mathrm{mW}$ (or $103 \mathrm{mV} / \mathrm{mW}$ after correction). The $3 \mathrm{~dB} f_{R}$ range of $2.21 \mathrm{GHz}$ indicates high sensitivity can be achieved at half of the frequency band. Or one can achieve high sensitivity of $17.3 \mathrm{mV} /$ $\mathrm{mW}$ (or $121 \mathrm{mV} / \mathrm{mW}$ after correction) and $3 \mathrm{~dB} f_{R}$ range of $1.6 \mathrm{GHz}$ at $\theta_{H}=75^{\circ}$ and $\delta_{H}=90^{\circ}$. The wide bandwidth of $7.5 \mathrm{GHz}$ offers some perspective for application in extended frequency band with moderate sensitivity. The sensitivity is expected to be further increased through improving the TMR ratio, reducing junction resistance for impedance matching, applying DC bias current, ${ }^{25}$ and exploiting materials with low saturation magnetization as free layer.

In summary, this work demonstrates that the bandwidth and sensitivity of spin-torque microwave detectors can be optimized through changing the direction of magnetic field. The influence of the angle and magnitude of hard-plane magnetic field on the $f_{R}$ and $V_{D C}$ is investigated. The $\mathrm{V}$-shape reliance of $f_{R}$ on $H_{\text {ext }}$ and on $\theta_{H}$ is qualitatively explicated by Kittel's ferromagnetic resonance model and quantitatively reproduced by an analytical model. Maximum $V_{D C}$ is observed at minimum $f_{R}$ due to the largest $\varphi$ at that point. The performance of the spin-torque diode detector is evaluated under three-dimensional magnetic field. Frequency bandwidth up to $9.8 \mathrm{GHz}$ or sensitivity up to $19.9 \mathrm{mV} / \mathrm{mW}$ (or $151 \mathrm{mV} / \mathrm{mW}$ after correction) can be achieved by applying magnetic field with different OOP angles. The sensitivity and frequency bandwidth can be flexibly tailored based on 
the specific requirements through tuning the OOP angle of the magnetic field.

This work was supported in part by the Seed Funding Program for Basic Research and Small Project Funding Program from the University of Hong Kong, ITF Tier 3 funding (ITS/203/14, ITS/104/13, and ITS/214/14), RGCGRF Grant (Nos. HKU 17210014 and HKU $704911 \mathrm{P}$ ), Innovation and Technology Fund Internship Programme (InP/182/14), and University Grants Committee of Hong Kong (Contract No. AoE/P-04/08).

${ }^{1}$ S. I. Kiselev, J. C. Sankey, I. N. Krivorotov, N. C. Emley, R. J. Schoelkopf, R. A. Buhrman, and D. C. Ralph, Nature 425, 380 (2003).

${ }^{2}$ A. A. Tulapurkar, Y. Suzuki, A. Fukushima, H. Kubota, H. Maehara, K. Tsunekawa, D. D. Djayaprawira, N. Watanabe, and S. Yuasa, Nature 438, 339 (2005).

${ }^{3}$ S. Hemour, Y. Zhao, C. H. P. Lorenz, D. Houssameddine, Y. Gui, C.-M. $\mathrm{Hu}$, and K. Wu, IEEE Trans. Microwave Theory Tech. 62, 965 (2014).

${ }^{4}$ X. Fan, R. Cao, T. Moriyama, W. Wang, H. W. Zhang, and J. Q. Xiao, Appl. Phys. Lett. 95, 122501 (2009).

${ }^{5}$ S. Miwa, S. Ishibashi, H. Tomita, T. Nozaki, E. Tamura, K. Ando, N. Mizuochi, T. Saruya, H. Kubota, K. Yakushiji, T. Taniguchi, H. Imamura, A. Fukushima, S. Yuasa, and Y. Suzuki, Nat. Mater. 13, 50 (2014).

${ }^{6}$ B. Fang, M. Carpentieri, X. Hao, H. Jiang, J. A. Katine, I. N. Krivorotov, B. Ocker, J. Langer, K. L. Wang, B. Zhang, B. Azzerboni, P. K. Amiri, G. Finocchio, and Z. Zeng, Nat. Commun. 7, 11259 (2016).

${ }^{7}$ C. Wang, Y.-T. Cui, J. Z. Sun, J. A. Katine, R. A. Buhrman, and D. C. Ralph, J. Appl. Phys. 106, 053905 (2009).

${ }^{8}$ T. Taniguchi and H. Imamura, Appl. Phys. Express 6, 053002 (2013).
${ }^{9}$ D. Bang, T. Taniguchi, H. Kubota, T. Yorozu, H. Imamura, K. Yakushiji, A. Fukushima, S. Yuasa, and K. Ando, J. Appl. Phys. 111, 07 C917 (2012).

${ }^{10}$ T. Taniguchi and H. Imamura, J. Appl. Phys. 114, 053903 (2013).

${ }^{11}$ X. Cheng, J. A. Katine, G. E. Rowlands, and I. N. Krivorotov, Appl. Phys. Lett. 103, 082402 (2013).

${ }^{12}$ C. Wang, Y. T. Cui, J. Z. Sun, J. A. Katine, R. A. Buhrman, and D. C. Ralph, Phys. Rev. B 79, 224416 (2009).

${ }^{13}$ W. X. Wang, Y. Yang, H. Naganuma, Y. Ando, R. C. Yu, and X. F. Han, Appl. Phys. Lett. 99, 012502 (2011).

${ }^{14}$ S. Ikeda, K. Miura, H. Yamamoto, K. Mizunuma, H. D. Gan, M. Endo, S. Kanai, J. Hayakawa, F. Matsukura, and H. Ohno, Nat. Mater. 9, 721 (2010).

${ }^{15}$ S. Yakata, H. Kubota, Y. Suzuki, K. Yakushiji, A. Fukushima, S. Yuasa, and K. Ando, J. Appl. Phys. 105, 07D131 (2009).

${ }^{16}$ R. Matsumoto, A. Chanthbouala, J. Grollier, V. Cros, A. Fert, K. Nishimura, Y. Nagamine, H. Maehara, K. Tsunekawa, A. Fukushima, and S. Yuasa, Appl. Phys. Express 4, 063001 (2011).

${ }^{17}$ S. Yuasa, Y. Suzuki, T. Katayama, and K. Ando, Appl. Phys. Lett. 87, 242503 (2005).

${ }^{18}$ C. Kittel, Phys. Rev. 71, 270 (1947).

${ }^{19}$ C. Kittel, Phys. Rev. 73, 155 (1948).

${ }^{20}$ Z. Zeng, K. H. Cheung, H. W. Jiang, I. N. Krivorotov, J. A. Katine, V. Tiberkevich, and A. Slavin, Phys. Rev. B 82, 100410 (2010).

${ }^{21}$ R. Matsumoto, H. Kubota, T. Yamaji, H. Arai, S. Yuasa, and H. Imamura, Jpn. J. Appl. Phys. 53, 123001 (2014).

${ }^{22}$ Y. V. Goryunov, N. N. Garifyanov, G. G. Khaliullin, I. A. Garifullin, L. R. Tagirov, F. Schreiber, T. Muhge, and H. Zabel, Phys. Rev. B 52, 13450 (1995).

${ }^{23}$ H. Suhl, Phys. Rev. 97, 555 (1955).

${ }^{24}$ W. Chen, G. de Loubens, J.-M. L. Beaujour, A. D. Kent, and J. Z. Sun, J. Appl. Phys. 103, 07A502 (2008).

${ }^{25}$ S. Ishibashi, K. Ando, T. Seki, T. Nozaki, H. Kubota, S. Yakata, H. Maehara, A. Fukushima, S. Yuasa, and Y. Suzuki, IEEE Trans. Magn. 47, 3373 (2011). 